\title{
The UAPO Solution for the Plane Wave Diffraction by a Resistive Half Plane: Validation Tests
}

\author{
Giovanni Riccio, Gianluca Gennarelli, Flaminio Ferrara, Claudio Gennarelli, \\ and Rocco Guerriero
}

\begin{abstract}
This article deals with the uniform asymptotic physical optics solution for the evaluation of the diffraction contribution related to a resistive half plane. A plane wave is considered at skew incidence with respect to the edge. The reflection and transmission coefficients for both the polarizations are determined accounting for the resistive boundary conditions on the screen and then used in the electric and magnetic equivalent surface currents at the beginning of the approach. The effectiveness of the proposed solution is here tested with the aid of a full-wave commercial software.
\end{abstract}

\section{Introduction}

The plane wave diffraction by a junction of equal resistive sheets having a nonplanar configuration in a two-dimensional propagation model was solved in [1] by using the uniform asymptotic physical optics (UAPO) approach. In particular, the incidence direction and the incident electric field were considered orthogonal and parallel to the junction, respectively. Comparisons with data resulting from a numerical technique based on the boundary element method assessed the effectiveness of the proposed solution for the investigated scalar problem. Unfortunately, two-dimensional propagation models have limited applications, and then diffraction problems need to be investigated when involving arbitrarily polarized electric fields at skew incidence with respect to the junction. Accounting for the features of the UAPO approach (see also [2-11] for a nonexhaustive list of references), the plane wave diffraction by an isolated resistive half plane must be first studied. Accordingly, the UAPO approach has been recently proposed for evaluating the diffracted field due

Manuscript received 20 December 2021.

Giovanni Riccio is with Department of Information and Electrical Engineering and Applied Mathematics, University of Salerno, via Giovanni Paolo II, 132, 84084 Fisciano, Salerno, Italy; e-mail: griccio@unisa.it.

Gianluca Gennarelli is with the Institute for Electromagnetic Sensing of the Environment, National Research Council, via Diocleziano, 328, 80124 Naples, Italy; e-mail: gennarelli.g@irea. cnr.it.

Flaminio Ferrara is with the Department of Industrial Engineering, University of Salerno, via Giovanni Paolo II, 132, 84084 Fisciano, Salerno, Italy; e-mail: flferrara@unisa.it.

Claudio Gennarelli is with the Department of Industrial Engineering, University of Salerno, via Giovanni Paolo II, 132, 84084 Fisciano, Salerno, Italy; e-mail: cgennarelli@unisa.it.

Rocco Guerriero is with the Department of Industrial Engineering, University of Salerno, via Giovanni Paolo II, 132, 84084 Fisciano, Salerno, Italy; e-mail: rguerrie@unisa.it. to an arbitrarily polarized plane wave at skew incidence with respect to the edge of a resistive half plane [12]. In this framework, the resistive boundary conditions have been used to determine the reflection and transmission coefficients for the electric field components parallel and perpendicular to the ordinary plane of incidence. It must be stressed that the knowledge of such coefficients is necessary not only for the computation of the geometrical optics (GO) field in the surrounding free space but also for the evaluation of the electric and magnetic physical optics equivalent surface currents in the radiation integral at the beginning of the UAPO approach. Obviously, only electric radiating source exists for a resistive half-plane. The resulting solution has been presented in matrix form according to the uniform geometrical theory of diffraction (UTD) [13], and its ability to compensate the jumps of the GO field at the shadow boundaries has been proved [12].

This article is devoted to assessing the reliability of the UAPO solution proposed in [12] to give accurate field values, and the RF module of Comsol Multiphysics is used to achieve this goal.

\section{The UAPO Solution}

Let us consider a thin lossy dielectric sheet characterized by the surface resistivity $R_{e}=-j \zeta_{0} /$ $k_{0} d\left(\varepsilon_{r}-1\right)$, where $d$ and $\varepsilon_{r}$ are the thickness and the relative permittivity of the screen, respectively, and $\zeta_{0}$ and $k_{0}$ are the impedance and the propagation constant, respectively, of the free space surrounding the planar structure. This last is truncated and approximated by a resistive half plane $S$ satisfying the following boundary conditions [14]:

$$
\begin{gathered}
\hat{n} \times\left.\left[\underline{E}^{+}-\underline{E}^{-}\right]\right|_{S}=0 \\
\hat{n} \times\left.[\hat{n} \times \underline{E}]\right|_{S}=-R_{e} \hat{n} \times\left.\left[\underline{H}^{+}-\underline{H}^{-}\right]\right|_{S}
\end{gathered}
$$

where the superscripts + and - identify the electric $(E)$ and magnetic $(H)$ fields on the upper and lower parts of $S$, respectively, and $\hat{n}$ is the unit vector normal to $S$.

As is well known, the resistive boundary conditions permit to model many simple structures, and analytical methods have been proposed in the literature to solve related diffraction problems. See [15-22] and references therein for limited and nonexhaustive bibliographies.

Accounting for the geometry in Figure 1, the analytical approach proposed in [12] has permitted to formulate the UAPO diffracted field in the UTD framework as follows: 


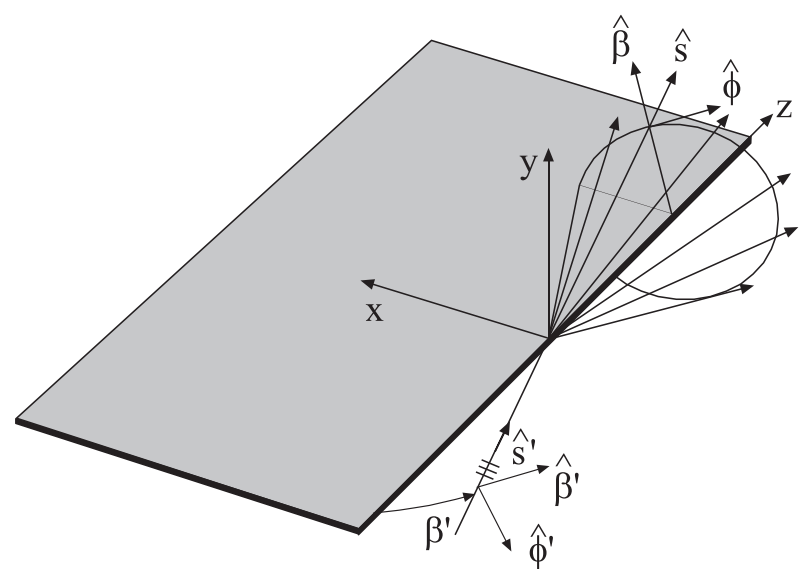

Figure 1. Geometry for the diffraction by a resistive half plane.

$$
\left(\begin{array}{c}
E_{\beta}^{d} \\
E_{\phi}^{d}
\end{array}\right)=\underline{\underline{D}} \frac{\exp \left(-j k_{0} s\right)}{\sqrt{s}}\left(\begin{array}{c}
E_{\beta^{\prime}}^{i} \\
E_{\phi^{\prime}}^{i}
\end{array}\right)
$$

where $s$ is the distance from the diffraction point to the observation point $P$ and

$$
\underline{\underline{D}}=I_{0}^{d} \underline{\underline{M}}
$$

The function $I_{0}^{d}$ includes the UTD transition function $F_{t}$ and results from a uniform asymptotic evaluation [12]:

$$
I_{0}^{d}=\frac{\exp (-j \pi / 4)}{2 \sqrt{2 \pi k_{0}}} \frac{F_{t}\left(2 k_{0} s \sin ^{2} \beta^{\prime} \cos ^{2}\left(\frac{\phi \pm \phi^{\prime}}{2}\right)\right)}{\sin ^{2} \beta^{\prime}\left(\cos \phi+\cos \phi^{\prime}\right)}
$$

Note that the sign $+(-)$ must be used if $0<\phi<\pi$ $(\pi<\phi<2 \pi)$.

The matrix $\underline{\underline{M}}$ in (4) contains the physical optics surface currents $\overline{\overline{a d}}$ opted as equivalent sources (only electric radiating sources exist for a resistive half plane) and can be so expressed [12]:

$$
\underline{\underline{M}}=\underline{\underline{M}}_{1} \underline{\underline{M}}_{2} \underline{\underline{M}}_{3} \underline{\underline{M}}_{4} \underline{\underline{M}}_{5}
$$

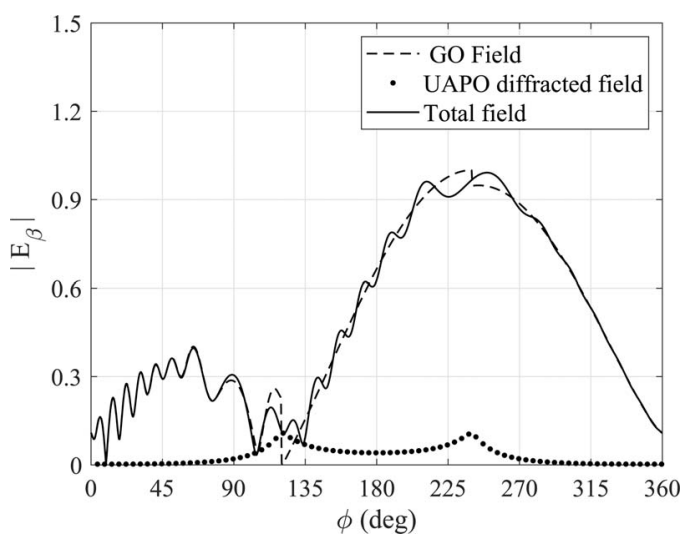

Figure 2. Co-polar component of the field when $E_{\beta^{\prime}}^{i}=1, E_{\phi^{\prime}}^{i}=0$ and $\beta^{\prime}=35^{\circ}, \phi^{\prime}=60^{\circ}$

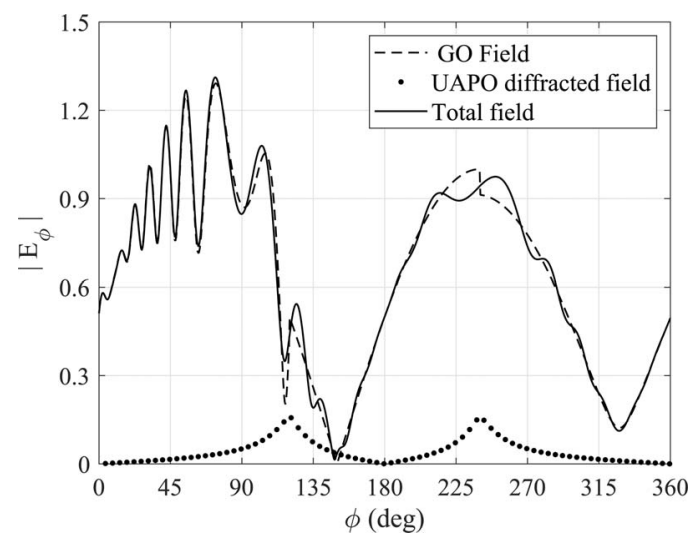

Figure 3. Co-polar component of the field when $E_{\beta^{\prime}}^{i}=0, E_{\phi^{\prime}}^{i}=1$ and $\beta^{\prime}=35^{\circ}, \phi^{\prime}=60^{\circ}$.

with

$$
\underline{\underline{M}}_{1}=\left(\begin{array}{ccc}
\cos \beta^{\prime} \cos \phi & \cos \beta^{\prime} \sin \phi & -\sin \beta^{\prime} \\
-\sin \phi & \cos \phi & 0
\end{array}\right)
$$

$\underline{\underline{M}}_{2}=\left(\begin{array}{cc}1-\sin ^{2} \beta^{\prime} \cos ^{2} \phi & -\sin \beta^{\prime} \cos \beta^{\prime} \cos \phi \\ -\sin ^{2} \beta^{\prime} \sin \phi \cos \phi & -\sin \beta^{\prime} \cos \beta^{\prime} \sin \phi \\ -\sin \beta^{\prime} \cos \beta^{\prime} \cos \phi & \sin ^{2} \beta^{\prime}\end{array}\right)$
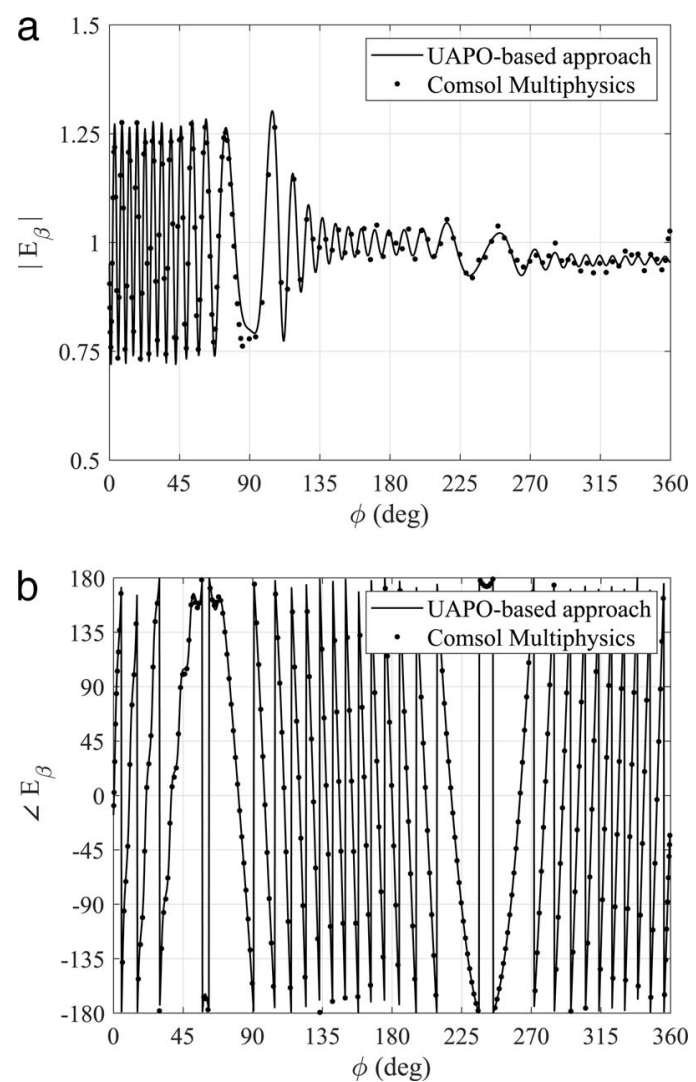

Figure 4. Co-polar component of the field when $E_{\beta^{\prime}}^{i}=1, E_{\phi^{\prime}}^{i}=0$ and $\beta^{\prime}=90^{\circ}, \phi^{\prime}=60^{\circ}$. (a) Magnitude of the total field. (b) Phase of the total field. 

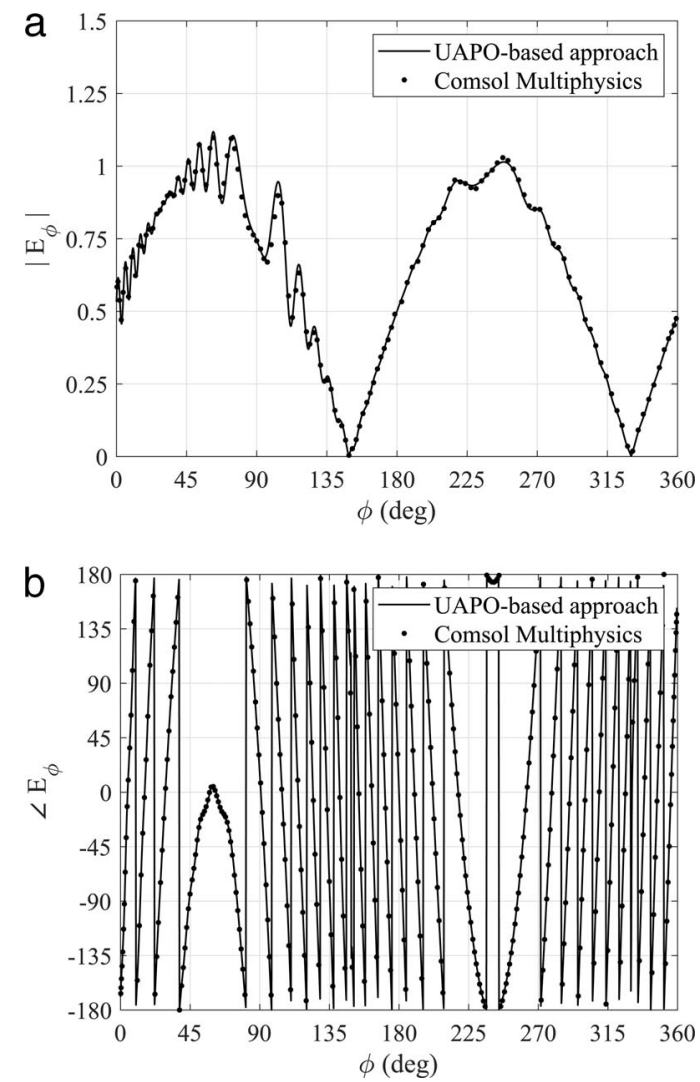

Figure 5. Co-polar component of the field when $E_{\beta^{\prime}}^{i}=0, E_{\phi^{\prime}}^{i}=1$ and $\beta^{\prime}=90^{\circ}, \phi^{\prime}=60^{\circ}$. (a) Magnitude of the total field. (b) Phase of the total field.

$$
\begin{aligned}
& \underline{\underline{M}}_{3}=\frac{1}{A\left(\beta^{\prime}, \phi^{\prime}\right)}\left(\begin{array}{cc}
-\cos \beta^{\prime} & -\sin \beta^{\prime} \cos \phi^{\prime} \\
-\sin \beta^{\prime} \cos \phi^{\prime} & \cos \beta^{\prime}
\end{array}\right) \\
& \underline{\underline{M}}_{4}=\left(\begin{array}{cc}
0 & \left(1-R_{\perp}-T_{\perp}\right) \sin \beta^{\prime} \sin \phi^{\prime} \\
1+R_{\|}-T_{\|} & 0
\end{array}\right) \\
& \underline{\underline{M}}_{5}=\frac{1}{A\left(\beta^{\prime}, \phi^{\prime}\right)}\left(\begin{array}{cc}
\cos \beta^{\prime} \sin \phi^{\prime} & \cos \phi^{\prime} \\
-\cos \phi^{\prime} & \cos \beta^{\prime} \sin \phi^{\prime}
\end{array}\right)
\end{aligned}
$$

with $A\left(\beta^{\prime}, \phi^{\prime}\right)=\sqrt{1-\sin ^{2} \beta^{\prime} \sin ^{2} \phi^{\prime}}$. The reflection $(R)$ and transmission $(T)$ coefficients for parallel $(\|)$ and perpendicular $(\perp)$ field components are determined by applying the boundary conditions (1) and (2) [12].

\section{Tests}

This section contains numerical examples concerning the plane wave scattering by a resistive half plane with $\varepsilon_{r}=2.56-j 0.023$ and $d=0.05 \lambda_{0}$, where $\lambda_{0}$ is the free-space wavelength. The data are collected on a circular path with radius equal to $7 \lambda_{0}$.
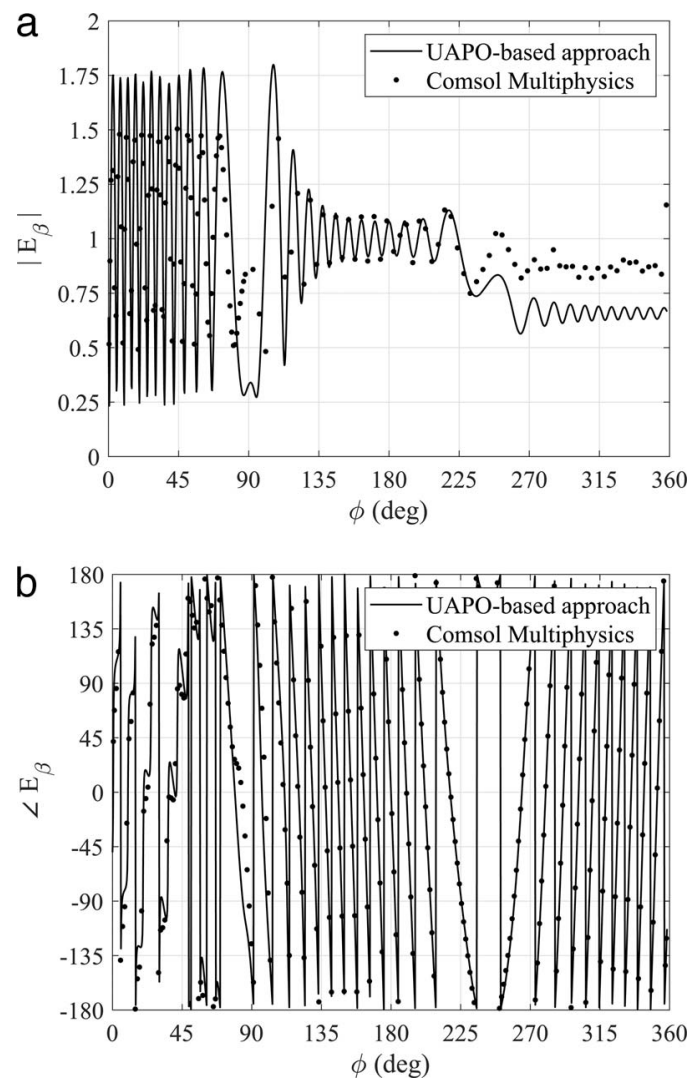

Figure 6. Co-polar component of the field when $E_{\beta^{\prime}}^{i}=1, E_{\phi^{\prime}}^{i}=0$, $\beta^{\prime}=90^{\circ}, \phi^{\prime}=60^{\circ}$ and $d=0.2 \lambda_{0}$. (a) Magnitude of the total field. (b) Phase of the total field.

Figures 2 and 3 contain the amplitudes of the GO field and the UAPO diffracted field as well as the total field obtained by adding them. Accounting for the incidence direction, the GO field possesses evident discontinuities at reflection and transmission boundaries as expected, whereas the continuity of the total field across such directions confirms the correct behavior of the UAPO solution in the UTD context. The total field values practically coincide with the GO field ones save for the angular sectors around the shadow boundaries, where the UAPO diffracted field is more relevant.

The following figures show the comparisons with the data obtained by using the RF module of Comsol Multiphysics. The normal incidence with respect to the edge of the resistive half plane is considered to reduce the needed computational effort. Magnitude and phase of $E_{\beta}$ are sketched in Figure 4 when $E_{\beta^{\prime}}^{i}=1, E_{\phi^{\prime}}^{i}=0$, and $\phi^{\prime}=60^{\circ}$. The UAPO-based values are in good agreement with the Comsol Multiphysics data, thus increasing the reliability of the proposed solution. A further assessment results from the comparisons in Figure 5, where magnitude and phase of $E_{\phi}$ are shown when $E_{\beta^{\prime}}^{i}=0, E_{\phi^{\prime}}^{i}=1$.

The accuracy of the UAPO-based total field decreases when increasing the thickness of the sheet. Many tests confirm this deterioration (see Figure 6 as 
example), and inaccurate results must be expected if $d$ is thick with respect to the wavelength since the approach does not consider the scattering contributions arising from the real geometry of the structure.

\section{Concluding Remarks}

The UAPO approach is conveniently exploited to tackle the plane wave diffraction by a resistive half plane when the incidence direction is oblique with respect to the edge. The closed-form analytic solution is UTD-like, compact, and user friendly. It permits to compensate the jumps of the GO field at the shadow boundaries, and its effectiveness is proved by means of comparisons with Comsol Multiphysics data. As expected, the accuracy of the UAPO-based total field decreases when increasing the thickness of the sheet.

\section{References}

1. C. Gennarelli, G. Pelosi, G. Riccio, and G. Toso, "Electromagnetic Scattering by Nonplanar Junctions of Resistive Sheets," IEEE Transactions on Antennas and Propagation, 48, 4, 2000, pp. 574-580.

2. G. Gennarelli and G. Riccio, "Diffraction by a Planar Metamaterial Junction With PEC Backing," IEEE Transactions on Antennas and Propagation, 58, 9, 2010, pp. 2903-2908.

3. G. Gennarelli and G. Riccio, "On the Accuracy of the UAPO Solution for the Diffraction by a PEC-DNG Metamaterial Junction," IEEE Antennas and Wireless Propagation Letters, 19, 4, 2020, pp. 581-585.

4. G. Gennarelli and G. Riccio, "High-Frequency Diffraction Contribution by Planar Metallic-DNG Metamaterial Junctions," International Journal of Microwave and Wireless Technologies, 12, 10, 2020, pp. 976-981.

5. G. Gennarelli and G. Riccio, "A Uniform Asymptotic Solution for Diffraction by a Right-Angled Dielectric Wedge," IEEE Transactions on Antennas and Propagation, 59, 3, 2011, pp. 898-903.

6. G. Gennarelli and G. Riccio, "Plane-Wave Diffraction by an Obtuse-Angled Dielectric Wedge," Journal of the Optical Society of America A, 28, 4, 2011, pp. 627-632.

7. G. Gennarelli and G. Riccio, "Diffraction by $90^{\circ}$ Penetrable Wedges With Finite Conductivity," Journal of the Optical Society of America A, 31, 1, 2014, pp. 2125 .

8. G. Gennarelli, M. Frongillo, and G. Riccio, "HighFrequency Evaluation of the Field Inside and Outside an Acute-Angled Dielectric Wedge," IEEE Transactions on Antennas and Propagation, 63, 1, 2015, pp. 374-378.

9. M. Frongillo, G. Gennarelli, and G. Riccio, "Diffraction by a Structure Composed of Metallic and Dielectric $90^{\circ}$ Blocks," IEEE Antennas and Wireless Propagation Letters, 17, 5, 2018, pp. 881-885.

10. M. Frongillo, G. Gennarelli, and G. Riccio, "Plane Wave Diffraction by Arbitrary-Angled Lossless Wedges: HighFrequency and Time-Domain Solutions," IEEE Transactions on Antennas and Propagation, 66, 12, 2018, pp. 6646-6653.

11. M. Frongillo, G. Gennarelli, and G. Riccio, "Useful Solutions for the Plane Wave Diffraction by a Configuration of Dielectric and Metallic Acute-Angled Wedges," International Journal on Communications Antenna and Propagation, 10, 2, 2020, pp. 68-75.

12. G. Riccio, G. Gennarelli, F. Ferrara, C. Gennarelli, and R. Guerriero, "The UAPO Solution for the Plane Wave Diffraction by a Resistive Half-Plane in the Case of Skew Incidence," XXXIVth URSI General Assembly and Scientific Symposium, Rome, Italy, August 2021, pp. 1-4.

13. R. G. Kouyoumjian and P. H. Pathak, "A Uniform Geometrical Theory of Diffraction for an Edge in a Perfectly Conducting Surface," Proceedings of the IEEE, 62, 11, 1974, pp. 1448-1461.

14. T. B. A. Senior and J. L. Volakis, Approximate Boundary Conditions in Electromagnetics, London, IEE, 1995.

15. T. B. A. Senior, "Diffraction by a Resistive Half Plane," Electromagnetics, 11, 2, 1991, pp. 183-191.

16. Y. Z. Umul, "Diffraction of Waves by a Resistive HalfPlane," Optics Communications, 323, 2014, pp. 6-12.

17. H. D. Basdemir, "PO and PTD Approach to the Diffraction Problem by a Resistive Half-Plane," Journal of Electromagnetic Waves and Applications, 28, 17, 2014, pp. 2188-2196.

18. Y. Z. Umul and U. Yalcin, "Diffraction Theory of Waves by a Resistive Surface," Progress in Electromagnetics Research B, 23, 2010, pp. 1-13.

19. Y. Z. Umul, "Diffraction of Plane Electromagnetic Waves by a Resistive Half-Screen for Skew Incidence," Journal of the Optical Society of America A, 37, 1, 2019, pp. 6369.

20. J. L. Volakis, "A Uniform Geometrical Theory of Diffraction for an Imperfectly Conducting Half-Plane," IEEE Transactions on Antennas and Propagation, 34, 2, 1986, pp. 172-180.

21. T. Lertwiriyaprapa, P. H. Pathak, and J. L. Volakis, "A UTD for the Radiation by Sources Near Thin Planar Positive or Negative Material Structures with a Discontinuity," Radio Science, 42, 6, 2007, RS6S18, doi: 10. 1029/2007RS003689.

22. T. B. A. Senior, "Some Problems Involving Imperfect Half Planes," in P. L. E. Uslenghi (ed.), Electromagnetic Scattering, New York, Academic Press, 1978, pp. 185219. 